\title{
Stable Profit Sharing in a Patent Licensing Game: General Bargaining Outcomes*
}

\author{
Naoki Watanabe ${ }^{\dagger}$ and Shigeo Muto ${ }^{\ddagger}$
}

April, 14, 2008

\begin{abstract}
By considering coalition structures formed by an external licensor of a patented technology and oligopolistic firms, we investigate licensing agreements that can be reached as bargaining outcomes under those coalition structures. The following results hold in a generalized patent licensing game. The core for a coalition structure is always empty, unless the grand coalition forms. We give a necessary and sufficient condition for the nonemptiness of the core (for the grand coalition). If the number of licensees that maximizes licensees' total surplus is greater than the number of existing non-licensees, each symmetric bargaining set for a coalition structure is a singleton, and the optimal number of licensees that maximizes the licensor's revenue is uniquely determined.
\end{abstract}

JEL Classification Numbers : C71; D45; D43

Keywords : licensing; coalition structure; bargaining set; core

${ }^{*}$ The authors wish to thank the chief editor, anonymous referees, and participants in the 10th DC (Japan), the 3rd ICMA, and the 17th Stony Brook conference for helpful comments and suggestions. Thanks are extended to Ryo Kawasaki for editing English. They are partially supported by the MEXT Grant-in-Aid for 21 Century COE Program, Grant-in-Aid 18730517 (Watanabe), and Grant-in-Aid 16310107 (Muto).

${ }^{\dagger}$ Department of Social Systems and Management, Graduate School of Systems and Information Engineering, University of Tsukuba, 1-1-1 Tennodai, Tsukuba, Ibaraki 3058573, Japan. E-mail: naoki50@sk.tsukuba.ac.jp.

${ }^{\ddagger}$ Department of Social Engineering, Graduate School of Decision Science and Technology, Tokyo Institute of Technology, 2-12-1 Oh-okayama, Meguro, Tokyo 152-8552, Japan. E-mail: muto@soc.titech.ac.jp 


\section{Introduction: Licensing and Bargaining}

This paper investigates licensing agreements that can be reached as results of negotiations among an external licensor of a patented technology and oligopolistic firms and aims to provide some implications on how many licenses the licensor should sell to firms through such negotiations.

Patent licensing problems in oligopolistic markets had been studied only by non-cooperative mechanisms; upfront fee or royalty in Kamien and Tauman $(1984,1986)$, and auction in Katz and Shapiro (1985, 1986). Specifying market structures, the subsequent papers have focused on the optimal licensing mechanisms that maximizes the licensor's revenue (e.g., Kamien, Oren, and Tauman (1992), Muto (1993), Sen (2005) and Sen and Tauman (2007)). On the other hand, licensing agreements are basically contract terms signed by licensors and licensees resulting from bargaining. From this practical viewpoint, we study patent licensing as bargaining outcomes. ${ }^{1}$

From a cooperative viewpoint, Tauman and Watanabe (2007, hereafter TW) recently gave an interpretation of the licensor's payoff in the noncooperative auction game. Their analysis was, however, limited to the study of asymptotic equivalence. In practice, each industry has a finite number of firms operating. For such an industry, Driessen, Muto, and Nakayama (1992, hereafter DMN) studied a cooperative game on information trading which is similar to patent licensing. Their analysis was, however, confined to payoff distributions in the grand coalition (i.e., every player shares the information). To consider the number of licenses that most benefits the licensor, we need to extend their approach. We hence study coalition structures formed by an external licensor of a patented technology and firms operating in oligopolistic markets and licensing agreements reached as bargaining outcomes under those coalition structures. For this purpose, we consider cooperative solutions for games with coalition structures, where no sidepayments among coalitions are allowed, as in Aumann and Drèze (1974) and the references therein. ${ }^{2}$

\footnotetext{
${ }^{1}$ See Sempere-Monerris and Vannetelbosch (2001) for a Cournot duopoly, although their analysis is limited to an extreme case where the licensor has full bargaining power.

${ }^{2}$ Thrall and Lucas (1963) should be noted as a related work.
} 
A key issue is how to define the worth of each coalition, i.e., characteristic function. Watanabe and Tauman (2003, hereafter WT) proposed a sophisticated definition under a subtle mixture of conflict and cooperation: licensees can form a cartel $S$ to enhance their oligopolistic power, whereas non-licensees may react also by forming cartels. Firms can merge or acquire the others freely. Thus, the licensees in $S$ might not merge into a single entity, but gather as a group of smaller subcartels in $S{ }^{3}$ In contrast to WT, we prohibit firms from forming any cartels in the market. This is the assumption under which we consider the same situation as those formalized with non-cooperative mechanisms in the literature. Further, we assume that every firm is licensed in the coalition including the licensor. In the spirit of DMN, firms in the coalition including the licensor are allowed to cooperate to maximize the total payoff of the coalition they belong to, so some firms agree not to be licensed via sidepayments. We do not allow firms to cooperate also in this way. (We discuss this point in Section 5.) This paper hence regards a coalition of a licensor and licensees as merely a group for negotiations on licensing issues.

Bargaining outcomes under coalition structures provide some implications on how many licenses the licensor should sell through negotiations. Our results are summarized as follows. (I) The core for a coalition structure is always empty, unless the grand coalition forms. (II) A necessary and sufficient condition for the nonemptiness of the core (for the grand coalition) is given as a simple expression. (III) The upper and lower bounds of the symmetric bargaining set for a coalition structures are given. (IV) If the number of licensees that maximizes their total surplus is greater than the number of existing non-licensees, each symmetric bargaining set for a coalition structure is a singleton and the optimal number of licensees that maximizes the licensor's revenue is uniquely determined. (V) When the grand coalition forms, the bargaining set coincides with the core, if the core is non-empty.

Our solutions are both defined in Aumann and Drèze (1974). The bar-

\footnotetext{
${ }^{3} \mathrm{TW}$ gave a simpler interpretation to this definition; within a coalition firms can merge into a single entity or can operate a few (or all) of them and shut down the others. See Section 5 for more detail.
} 
gaining set for a coalition structure is due to Davis and Maschler (1967). ${ }^{4}$ The sharp result (IV) is concisely derived from (III). We show in the Appendix that the complete characterization of the bargaining set for a coalition structure would not give any tighter predictions in comparison to those shown by other propositions. In the bargaining set family, we could have chosen the kernel or nucleolus (for a coalition structure) as our solution ${ }^{5}$. They are, however, based on the cardinal measure of complaints of coalitions to proposed payoffs, so they represent "ethical" standards to possible bargaining outcomes. The "stable" profit sharing is to be the amount the players can obtain when no convincing objection can be made by any players. Note that, in this paper, neither objection nor counter objection is required to be stable in any sense. There are many variants of the bargaining set, some of which require that counter objections match several simultaneous objections. For each coalition structure, the bargaining set applied in this paper contains those bargaining sets as well as the core. ${ }^{6}$ We show that such a bargaining set is a singleton for some coalition structures (result (IV)).

In the literature, linear demand and cost functions, Cournot or Bertrand oligopoly, cost-reducing or quality-improving technology, perfect or imperfect patent protection are assumed. ${ }^{7}$ Instead, we analyze a much less specified model, i.e., a generalized patent licensing game. We retain the traditional assumption that all firms have an identical production technology before a patented technology is licensed and that every licensee firm of the patented technology can use the same technology. We hence confine our main concern to symmetric payoffs to licensee firms.

The outline of this paper is as follows. For a better understanding of our generalization of licensing games, Section 2 gives a linear example that has been mainly analyzed in the literature. The recent literature is also reviewed in detail there. Section 3 formalizes the general licensing game and defines

\footnotetext{
${ }^{4}$ Aumann and Maschler (1964) originally defined the bargaining set for the grand coalition, but did not consider the bargaining set for a coalition structure explicitly.

${ }^{5}$ See also Aumann and Drèze (1974). The original definitions are due to Davis and Maschler (1965) and Schmeidler (1969), respectively.

${ }^{6}$ Many of those variants are empty for some games as well as the core.

${ }^{7}$ Kamien, Tauman, and Zhang (1988) studied patent licensing by means of fees for a new product. Muto (1987) considered a situation under resale-free.
} 
the solutions. Section 4 provides our main results and discusses the optimal number of licenses the licensor should sell through negotiations. Section 5 discusses the related works including characteristic functions that appeared in TW and DMN, where the other solutions are also briefly mentioned.

\section{A Linear Example: Literature Review}

In this section, we provide a linear patent licensing game that has been analyzed in the literature and explain how and why we generalize it. In Section 4 , we refer to this example. The theoretical developments in the literature that are referred in Section 1 are also reviewed here.

There are $n$ firms, $2 \leq n<\infty$, each producing an identical commodity. The production cost per unit of output is $c(>0)$. Let $q_{i}$ be the output level produced by firm $i$. The market of the commodity is cleared at the price $p=\max \left(a-\sum_{i \in N} q_{i}, 0\right)$, where $a$ is a constant and $c<a<\infty .{ }^{8}$

An agent has the patent of a technology which reduces the unit cost of production from $c$ to $c-\epsilon(>0)$. The profit of firm $i$ is $p q_{i}-(c-\epsilon) q_{i}$ if $i$ has an access to the patented technology (firm $i$ is called a licensee), while it is $p q_{j}-c q_{j}$ if $j$ has no access to that technology (firm $j$ is called a non-licensee). The agent has no production ability but shares the profits of licensees (the agent is called an external licensor).

Suppose that $s$ firms are licensed at the rates of upfront fee $F$ and royalty $r(\leq \epsilon)$ per unit of output. The upfront fee is paid to the licensor when the license is purchased. Assume that the patented technology is protected perfectly. Firms compete $\grave{a}$ la Cournot (i.e., in quantities) in the market, knowing which firms are licensed or not. Finally, the royalty is paid. Let $W(s, \delta)$ and $L(s, \delta)$ denote the equilibrium gross profit of each licensee and that of each non-licensee respectively, where $\delta=\epsilon-r$. We write $L(0, \delta)$ as $L(0)$. Let $\hat{s}:=(a-c) / \delta$. Then

$$
W(s, \delta)=\left\{\begin{array}{cc}
((a-c+(n-s+1) \delta) /(n+1))^{2} & \text { if } s<\hat{s} \\
((a-c+\delta) /(s+1))^{2} & \text { if } s \geq \hat{s}
\end{array}\right.
$$

\footnotetext{
${ }^{8}$ This condition guarantees that the supply of the product is socially desirable.
} 


$$
L(s, \delta)=\left\{\begin{array}{cl}
((a-c-s \delta) /(n+1))^{2} & \text { if } s<\hat{s} \\
0 & \text { if } s \geq \hat{s},
\end{array}\right.
$$

which are summarized in the following order:

$$
\begin{gathered}
W(1, \delta)>\cdots>W(s, \delta)>\cdots>W(n, \delta)>L(0)>\cdots>L(s, \delta) \\
>\cdots>L(\hat{s}-1, \delta)>L(\hat{s}, \delta)=\cdots=L(n-1, \delta)=0 .
\end{gathered}
$$

Regardless of the differences in the gross equilibrium profits of firms, this order is preserved in various patent licensing games with other market structures including Bertrand competition (with homogeneous good or differentiated goods) and markets for new products.

In the literature, the amount $F$ of upfront fee or the royalty rate $r$ is determined so as to maximize the revenue of the licensor. After the seminal papers noted in Section 1, Kamien, Oren, and Tauman (1992) showed that in a Cournot competition for a homogeneous good it is never optimal for the external licensor to license a cost-reducing technology by means of royalty only. Muto (1993) found that in a Bertrand duopoly with differentiated commodities there are cases where it is optimal for an external licensor to license by means of royalty only. For a cost reducing technology in a Cournot industry, Sen (2005) reconsidered this traditional argument on fee versus royalty and Sen and Tauman (2007) found the optimal combination of upfront fee and royalty. ${ }^{9}$ Tauman and Watanabe (2007) showed that in a Cournot market, when the number of firms is large, the Shapley value of the licensor in a cooperative approach approximates his payoff in the non-cooperative auction game traditionally studied. ${ }^{10}$

All these results are, however, derived under perfect patent protection. By the nature of patented technologies, the spillover (including piracy and resale) to non-licensees is inevitable. The above order of the gross equilibrium profits may not be necessarily preserved in the presence of some patterns of spillover. Moreover, it is difficult to predict which pattern of spillover actually occurs. Thus, we use neither the values of the equilibrium

\footnotetext{
${ }^{9}$ They also considered the case of incumbent licensor who has production ability.

${ }^{10} \mathrm{It}$ is remarkable that the two approaches asymptotically coincide, because the patent holder does not have full bargaining power in the cooperative approach and the Shapley value measures the fair contribution of the patent holder to the total industry profit.
} 
gross profits of firms nor the above order of them, and use only the following:

$$
W(s)>L(0) \forall s=1, \ldots, n, \quad L(0)>L(s) \forall s=1, \ldots, n-1 .
$$

This is our generalization of patent licensing games that appeared in the literature. We allow sidepayments, so there is no substantial difference of payments between before and after the market competition. We focus on the total payment to the licensor, so let $\delta=\epsilon$ in $W(s)$ and $L(s)$. We briefly mention limited sidepayments in Subsection 5.3.

\section{A Generalized Patent Licensing Game}

\subsection{A Game with a Coalition Structure}

Let $N=\{1, \ldots, n\}$ be a set of identical firms (producing a homogeneous good or differentiated goods). An external licensor (not being a producer), called player 0 , has a patent of a (cost-reducing or quality-improving) technology. Thus, the set of players is $\{0\} \cup N$. Each non-empty subset of $\{0\} \cup N$ is called a coalition.

The game has three stages. (i) At the beginning, the licensor selects a subset $S \subseteq N$ of firms and invites them to the negotiation on license issues. Firms that belong to $N \backslash S$ cannot participate in that negotiation, so they are not licensed. (ii) At this stage, every firm in $S$ negotiates with the licensor over how much it should pay to the licensor. It is assumed that all the firms in $S$ that were invited to bargain will buy a license, thus focusing solely on the fees paid to the licensor. (Even if firms in $S$ could choose whether or not to buy the license, we would retain the same propositions due to the solution concepts we apply to this model.) All players in $\{0\} \cup S$ can communicate within $\{0\} \cup S$. Each non-licensee firms is not allowed to communicate with any players. The payment is made before the next competition stage. (iii) Firms compete in the market, knowing which firms are licensed or not. (The market can be Cournot or Bertrand competition.) They are not allowed to form a cartel both in production and in the market.

Remark 1: No negotiation process is specified at stage (ii). A conference might be held by all members of $\{0\} \cup S$, or the licensor might negotiate 
with each firm in $S$ on a one-by-one basis. More important is that players in $\{0\} \cup S$ can communicate among themselves, whereas non-licensees cannot observe how the negotiations run. This is a difference from the traditional non-cooperative models, where firms cannot communicate with any others.

We intend to provide some implications on the optimal number of licenses according to the analysis for each set $S$ of firms the licensor chooses as licensees at invitation stage (i). Given such a set $S$ of licensees, our solutions, defined in the next subsection, prescribe bargaining outcomes on the payment to the licensor in the situation noted in remark 1. Let us first define a game $\left(\{0\} \cup N, v, P^{S}\right)$ with coalition structure below.

Let $s=|S|$ for each $S \subseteq N(|S|=0$ if $S=\emptyset)$. When $s$ firms hold the license, let $W(s)$ and $L(s)$ denote the equilibrium gross profits of a licensee and a non-licensee, respectively, at competition stage (iii). We require only

$$
W(s)>L(0) \forall s=1, \ldots, n, \quad L(0)>L(s) \forall s=1, \ldots, n-1,
$$

as noted in Section 2. ${ }^{11}$ Given this feature at stage (iii), let us next formalize negotiation stage (ii). For any $S \subseteq N$ with $S \neq \emptyset$, let $\min _{r=|R|, R \subseteq N \backslash S} L(r)=$ $L(\rho(s))$. As noted in Section 1, firms are not allowed to form a cartel at competition stage (iii). Further, we prohibit another type of cooperation via sidepayments at negotiation stage (ii), i.e., every firm in coalition $\{0\} \cup S$ is licensed. The characteristic function $v: 2^{\{0\} \cup N} \rightarrow \mathbb{R}$ is then defined by

$$
\begin{aligned}
& v(\{0\})=v(\emptyset)=0, \quad v(\{0\} \cup S)=s W(s), \\
& v(S)=s L(\rho(s)),
\end{aligned}
$$

where $v\left(S^{\prime}\right)$ represents the worth of a coalition $S^{\prime} \subseteq\{0\} \cup N$. The external licensor can gain nothing without selling the patented technology because he is not a producer. $v(\{0\} \cup S)=s W(s)$, because the total equilibrium gross profits of licensees in $S$ is $s W(s)$. The total equilibrium gross profits $v(S)$ that the firms in $S$ can guarantee for themselves even in the following pessimistic anticipation is $s L(\rho(s))$; they anticipate that the other $\rho(s)(\leq n-s)$ firms are licensed when firms in $S$ jointly break off the negotiations and they

\footnotetext{
${ }^{11}$ See Nakayama and Quintas (1991) for an asymmetric treatment of the firms' profits at competition stage (iii).
} 
cannot predict which coalition structure newly forms. ${ }^{12}$ This anticipation is made at negotiation stage (ii), but not necessarily implemented at competition stage (iii).

Given a set $S \subseteq N$ of licensees, the permissible coalition structure is $P^{S}=\left[\{0\} \cup S,\{\{j\}\}_{j \in N \backslash S}\right]$, because any players in $\{0\} \cup S$ can communicate among themselves but non-licensees are not allowed to communicate with any players, as noted in remark 1 . The set of imputations for a permissible coalition structure $P^{S}$ is defined as

$$
\begin{aligned}
X^{S}=\{x= & \left(x_{0}, x_{1}, \cdots, x_{n}\right) \in \mathbb{R}^{n+1} \mid x_{0}+\sum_{i \in S} x_{i}=s W(s), \\
& \left.x_{0} \geq 0, x_{i} \geq L(\rho(1)) \forall i \in S, x_{j}=L(s) \forall j \in N \backslash S\right\} .
\end{aligned}
$$

We hereafter consider only a subset $S$ of licensees with $S \neq \emptyset$, because the licensor can guarantee the payoff zero by itself, i.e., $x_{0} \geq v(\{0\})=0$ in $X^{S}$ for any $S \subseteq N$. Let $\left(\{0\} \cup N, v, P^{S}\right)$ be a game with a coalition structure $P^{S}$, where all the vector of payoffs should be in $X^{S}$. This is the game formalized as negotiation stage (ii). The solutions are defined in the next subsection.

\subsection{The Solutions for Stable Profit Sharing}

We apply two solutions to the game with a coalition structure defined above in order to figure out the bargaining outcomes as stable profit sharing.

First, the core for a coalition structure $P^{S}$ (of a game $\left(\{0\} \cup N, v, P^{S}\right)$ ) is the subset of $X^{S}$ and defined as

$$
C^{S}=\left\{x \in X^{S} \mid \sum_{i \in T} x_{i} \geq v(T) \forall T \subseteq\{0\} \cup N, T \cap(\{0\} \cup S) \neq \emptyset\right\}
$$

We simply call $C^{N}$ the core. Note that it is easy to show that, for any $S$, $C^{S}$ can be rewritten as $\left\{x \in X^{S} \mid \sum_{i \in T} x_{i} \geq v(T) \forall T \subseteq\{0\} \cup N\right\}$, which is the one defined in Aumann and Drèze (1974). We specified the set $X^{S}$ of imputations for a permissible coalition structure $P^{S}$ taking into account competitive stage (iii).

Next, we define the bargaining set for a coalition structure. Let $i, j \in$ $\{0\} \cup S$ and $x \in X^{S}$. We say that $i$ has an objection $(y, T)$ against $j$ at $x$

\footnotetext{
${ }^{12}$ The definition of $v(S)$ from this pessimistic viewpoint plays no important role in the proofs of our propositions.
} 
if $i \in T, j \notin T, T \subseteq\{0\} \cup N, y_{k}>x_{k}$ for any $k \in T$, and $\sum_{k \in T} y_{k} \leq v(T)$, and that $j$ has a counter objection $(z, R)$ to $i$ 's objection $(y, T)$ if $j \in R$, $i \notin R, R \subseteq\{0\} \cup N, z_{k} \geq x_{k}$ for any $k \in R, z_{k} \geq y_{k}$ for any $k \in R \cap T$, and $\sum_{k \in R} z_{k} \leq v(R)$. We say that $i$ has a valid objection $(y, T)$ against $j$ at $x$ if $(y, T)$ is not countered. The bargaining set for a coalition structure $P^{S}$ (of a game $\left.\left(\{0\} \cup N, v, P^{S}\right)\right)$ is also a subset of $X^{S}$ and defined as

$$
M^{S}=\left\{x \in X^{S} \mid \text { no player in }\{0\} \cup S \text { has a valid objection at } x\right\} .
$$

We simply call $M^{N}$ the bargaining set. This is the definition in Aumann and Drèze (1974), which is originally defined in Davis and Maschler (1967). ${ }^{13}$

In our model, any firms are identical before a patented technology is licensed, and the licensees are provided the same patented technology. So, we mainly consider symmetric payoffs to licensees. The symmetric solutions are defined as

$$
\tilde{C}^{S}=C^{S} \cap \tilde{X}^{S}, \quad \tilde{M}^{S}=M^{S} \cap \tilde{X}^{S},
$$

where $\tilde{X}^{S}=\left\{x \in X^{S} \mid x_{i}=x_{j}=\bar{x} \forall i, j \in S\right\}$.

Remark 2: In the definitions of our solutions, the firms that do not belong to $S$ cannot participate in the negotiations on license issues, but play a relevant role in determining the outside options of negotiators in $\{0\} \cup S$.

\section{The Main Results}

\subsection{The Core for a Coalition Structure}

By the definitions, $C^{S} \subseteq M^{S}$ for any $S \subseteq N$, if they are non-empty. If $C^{S}$ is non-empty for any $S \subseteq N$, we can predict stable profit sharing as bargaining outcomes more clearly by $C^{S}$. We hence begin with considering the stronger solution $C^{S}$. Unfortunately, however, our answer is negative. To show this, the next lemma is helpful. This lemma is intuitively obvious by the definition of $C^{S}$, so the proof is shown in the Appendix.

Lemma 1 For any $S \subseteq N$, if $C^{S} \neq \emptyset$, then there exists an $x \in \tilde{C}^{S}$.

\footnotetext{
${ }^{13}$ Some similar results can be obtained with other solutions such as the strong equilibrium and the coalition-proof Nash equilibrium. See Muto (1990) for the reference.
} 
Proposition $1 C^{S}=\emptyset$ if $S \neq N$.

Proof: We first show that $\tilde{C}^{S}=\emptyset$ if $S \neq N$. Suppose $\tilde{C}^{S} \neq \emptyset$. Take $x \in \tilde{C}^{S}$ with $x_{i}=\bar{x}$ for any $i \in S$. If $\bar{x} \leq L(0), \sum_{i \in N} x_{i}=s \bar{x}+(n-$ $s) L(s)<n L(0)=v(N)$ because $L(0)>L(s)=x_{j}$ for any $j \in N \backslash S$. Hence, $\bar{x}>L(0)$. Next, take a coalition $\{0\} \cup T$ such that $|T|=|S|$, $T \subseteq N \backslash S$ if $|S| \leq n / 2$ and $T \supseteq N \backslash S$ if $|S|>n / 2$. Let $t=|T|$. Then, $x_{0}+\sum_{i \in T} x_{i}<s W(s)=t W(t)$, because $x_{0}+s \bar{x}=s W(s)$ and $\bar{x}>L(0)>L(s)$. This contradicts $x \in \tilde{C}^{S}$. Finally, $\tilde{C}^{S}=\emptyset$ implies $C^{S}=\emptyset$ by Lemma 1.

Let $s^{*}$ be the number of licensees that maximizes their total surplus, i.e., $s^{*}\left(W\left(s^{*}\right)-L(0)\right) \geq s(W(s)-L(0))$ for any $s=1, \ldots, n$. The first statement of the next proposition provides a necessary and sufficient condition for the nonemptiness of $C^{N}$, which is similar to the one in a production economy formalized in Shapley and Schubik (1967). ${ }^{14}$

Proposition $2 C^{N} \neq \emptyset$ if and only if $s^{*}=n$. When $s^{*}=n, x \in \tilde{C}^{N}$ is characterized by $x_{0}+n \bar{x}=n W(n), 0 \leq x_{0} \leq n(W(n)-L(0))$ and $L(0) \leq \bar{x} \leq \min _{s: s \neq n}(n W(n)-s W(s)) /(n-s)$.

Proof: We begin with a necessary and sufficient condition for $C^{N} \neq \emptyset$.

(only if) Suppose that $s^{*}<n$. If $C^{N} \neq \emptyset$, there is an $x \in \tilde{C}^{N} \neq \emptyset$ by Lemma 1. Let $x_{i}=\bar{x}$ for any $i \in N$ and $x_{0}=n W(n)-n \bar{x}$. Then,

$$
\bar{x} \geq L(0) \quad \text { and } \quad x_{0}+s \bar{x} \geq s W(s), s=0,1, \cdots, n-1 .
$$

Letting $s=s^{*}$ in the latter condition of (2) gives $n W(n)-n \bar{x}+s^{*} \bar{x} \geq$ $s^{*} W\left(s^{*}\right)$ or $\left(n-s^{*}\right) \bar{x} \leq n W(n)-s^{*} W\left(s^{*}\right)$. By the former condition of (2), $\left(n-s^{*}\right) L(0) \leq n W(n)-s^{*} W\left(s^{*}\right)$ or $s^{*}\left(W\left(s^{*}\right)-L(0)\right) \leq n(W(n)-L(0))$, contradicting that $n>s^{*}=\arg \max _{s=1, \ldots, n} s(W(s)-L(0))$.

(if) Take $x$ such that

$$
x_{i}= \begin{cases}n(W(n)-L(0)) & \text { if } i=0 \\ L(0) & \text { if } i \in N .\end{cases}
$$

\footnotetext{
${ }^{14}$ See Muto, Nakayama, Potters and Tijs (1989) for more general analysis.
} 
Because $s^{*}=n$, it is easily shown that $x \in \tilde{C}^{N}$.

When $s^{*}=n$, the system (2) of inequalities implies $L(n-s) \leq \bar{x} \leq$ $(n W(n)-s W(s)) /(n-s)$ for any $s$. It suffices to show that $L(0) \leq \bar{x}$. Suppose $L(0)>\bar{x}$. Then, a licensor $i \in N$ can make an objection $(y, N)$, where $y_{i}=L(0)$ for any $i \in N$. Because $\sum_{i \in N} x_{i}<v(N)=n L(0)$, any $x$ with $L(0)>\bar{x}$ is not in $\tilde{C}^{N}$, contradiction.

Remark 3: In the linear example described in Section 2, it is easy to confirm that $s^{*}<n$ and thus $C^{S}=\emptyset$ for any permissible coalition structures $P^{S}$. Even in a linear environment, however, Watanabe and Muto (2006) showed a case where $C^{N} \neq \emptyset$ in a Bertrand duopoly with differentiated goods analyzed in Muto (1993).

\subsection{The Bargaining Set for a Coalition Structure}

When the solution is empty at negotiation stage (ii), we cannot answer our question on how many licenses the licensor should sell to firms through negotiations. Propositions 1 and 2 suggest that we consider a weaker solution $M^{S}$ to predict stable profit sharing in our patent licensing game. In contrast to the core for a coalition structure, the bargaining set for a coalition structure is always non-empty, which was shown by Peleg (1967).

Let us hereafter confine our attention to $\tilde{M}^{S}$. It is not unnatural to focus on the symmetric payoffs to identical licensees, because they have the same technologies both before and after licensed. Then, it suffices to examine objections and counter objections of the licensor and a licensee $i \in S$. Maschler and Peleg (1966) indirectly implies that $\tilde{M}^{S} \neq \emptyset$ for any $S \subseteq N .^{15}$

We first show the upper and lower bounds of $x_{0}$ in $\tilde{M}^{S}$, where $S \neq N$, in the next proposition.

Proposition 3 Let $x \in \tilde{M}^{S}$ with $S \neq N$. Then, we have the following. (a) If $1 \leq s \leq n / 2$ and $s(W(s)-L(0))>(n-s)(W(n-s)-L(0))$, then

$$
s\left(W(s)-W^{*}\right) \leq x_{0} \leq s^{*}\left(W\left(s^{*}\right)-L(0)\right),
$$

\footnotetext{
${ }^{15}$ They actually showed that the kernels for coalition structures are all non-empty, which allocate symmetric payoffs to identical players.
} 
where $W^{*}$ be the minimum of $W$ that satisfies the inequality $s(W(s)-W) \leq$ $(n-s)(W(n-s)-L(0))$. Note that $W^{*}>L(0)$.

(b) If $n / 2 \leq s<n$ or $s(W(s)-L(0)) \leq(n-s)(W(n-s)-L(0))$, then

$$
s(W(s)-L(0)) \leq x_{0} \leq s^{*}\left(W\left(s^{*}\right)-L(0)\right) .
$$

The proof of Proposition 3 consists of many lemmas. Before proceeding to their formal proofs, we here explain some of them intuitively. Let $s$ firms in $S$ be licensed. Lemmas 2 and 3 are interpreted as follows.

By the definition of $s^{*}, s(W(s)-L(0)) \leq s^{*}\left(W\left(s^{*}\right)-L(0)\right)$. Hence, when the licensor obtains more than $s^{*}(W(s)-L(0))$, each of $s$ licensees obtains less than $L(0)$. Then, all the licensees and non-licensees can be better off by forming a grand coalition $N$ and distributing $L(0)$ equally to each member of this objecting coalition. The licensor cannot make any counter objections against anyone in $N$. Therefore, $s^{*}\left(W\left(s^{*}\right)-L(0)\right)$ is the upper bound of the licensor's share of the total profit $s W(s)$ of coalition $\{0\} \cup S$. This is the intuition of Lemma 2.

On the other hand, when the licensor obtains less than $s(W(s)-L(0))$, each licensee in $S$ obtains more than $L(0)$. Then, the licensor can be better off by forming another coalition with all the $n-s$ non-licensees and $2 s-n$ licensees and dividing the total gain $(n-s)(\bar{x}-L(0))$ from non-licensees equally as an additional payoff to each member of this objecting coalition. No licensee in $S$ can make any counter objections against the licensor. This is the intuition of Lemma 3. Lemmas 4 and 5 amend the case where the condition $2 s-n>0$ required in Lemma 3 is not satisfied.

Proof: The proposition is proved by the following 4 lemmas. Lemma 2 suggests the upper bound of $x_{0}$. Under the corresponding conditions, Lemmas 3,4 and 5 suggest the upper bound of $\hat{x}$, which implies the lower bound of $x_{0}$. Let $x_{i}=\bar{x}$ for any licensees $i \in S$.

Lemma 2 For any $S \subseteq N$, if $x \in \tilde{M}^{S}$ then $x_{0} \leq s^{*}\left(W\left(s^{*}\right)-L(0)\right)$.

Proof : Let $x \in \tilde{M}^{S}$. Suppose $x_{0}>s^{*}\left(W\left(s^{*}\right)-L(0)\right)$. By the definition of $s^{*}, \bar{x}=\left(s W(s)-x_{0}\right) / s<\left(s W(s)-s^{*}\left(W\left(s^{*}\right)-L(0)\right)\right) / s \leq L(0)$. Take an objection $(y, N)$ of $i \in S$ against the licensor in $x$ with $y_{k}=L(0)$ for any $k \in$ 
$N$. If the licensor had a counter objection $(z,\{0\} \cup T)$ to the objection with $z_{0} \geq x_{0}>s^{*}\left(W\left(s^{*}\right)-L(0)\right)$ and $z_{k} \geq y_{k}=L(0)$ for any $k \in T$, it should be $z_{0}+\sum_{k \in T} z_{k}>s^{*}\left(W\left(s^{*}\right)-L(0)\right)+t L(0) \geq t W(t)$ by the definition of $s^{*}$, where $t=|T|$. Hence, no counter objection can be made, contradicting that $x \in \tilde{M}^{S}$.

Lemma 3 Suppose $n / 2 \leq s<n$. If $x \in \tilde{M}^{S}$, then $\bar{x} \leq L(0)$.

Proof: Suppose $\bar{x}>L(0)$. Take an objection $(y,\{0\} \cup T)$ of the licensor against firm $i \in S$ in $x$ such that $|T|=|S|, T \supseteq N \backslash S$ and

$$
y_{k}= \begin{cases}x_{0}+\epsilon & \text { if } k=0 \\ \bar{x}+\epsilon & \text { if } k \in T \cap S \\ L(0)+\epsilon & \text { if } k \in T \cap(N \backslash S),\end{cases}
$$

where $\epsilon=(n-s)(\bar{x}-L(0)) /(s+1)>0$ and $s=|S|$. Note that

$$
\begin{aligned}
y_{0}+\sum_{k \in T} y_{k} & =x_{0}+(2 s-n) \bar{x}+(n-s) L(0)+(s+1) \epsilon \\
& =x_{0}+(2 s-n) \bar{x}+(n-s) L(0)+(n-s)(\bar{x}-L(0)) \\
& =x_{0}+s \bar{x}=s W(s) .
\end{aligned}
$$

Because $y_{k}>L(0)$ for any $k \in T$ and $x_{k}=\bar{x}>L(0)$ for any $k \in N \backslash T$, any firms $i \in S$ has no counter objection to the objection, contradicting that $x \in \tilde{M}^{S}$.

When each of $s$ licensees obtains $\bar{x}=L(0)$ as the upper bound of its payoff, the licensor's profit sharing is at least $s(W(s)-s L(0))$. So is in the next case.

Lemma 4 Suppose $1 \leq s \leq n / 2$ and $s(W(s)-L(0)) \leq(n-s)(W(n-s)-$ $L(0))$. Then, if $x \in \tilde{M}^{S}$ then $\bar{x} \leq L(0)$

Proof : Let $x \in \tilde{M}^{S}$. Suppose $\bar{x}>L(0)$. Then $x_{0}<s(W(s)-L(0))$, because $x_{0}+s \bar{x}=s W(s)$. Take an objection $(y,\{0\} \cup(N \backslash S))$ of the licensor against firm $i \in S$ in $x$ such that

$$
y_{k}= \begin{cases}(n-s)(W(n-s)-L(0)) & \text { if } k=0 \\ L(0) & \text { if } k \in N \backslash S .\end{cases}
$$


Because $y_{k}=L(0)$ for any $k \in N \backslash S$ and $x_{k}=\bar{x}>L(0)$ for any $k \in S$, there is no counter objection to the objection, contradicting that $x \in \tilde{M}^{S}$.

In the next case, each of $s$ licensees obtains at most $W^{*}$, so the licensor obtains at least $s\left(W(s)-W^{*}\right)$.

Lemma 5 Suppose $1 \leq s \leq n / 2$ and $s(W(s)-L(0))>(n-s)(W(n-$ $s)-L(0))$. Let $W^{*}$ be the minimum of $W$ that satisfies $s(W(s)-W) \leq$ $(n-s)(W(n-s)-L(0))$. Then, if $x \in \tilde{M}^{S}$ then $\bar{x} \leq W^{*}$

Proof: Suppose $\bar{x}>W^{*}$. Then $x_{0}<s\left(W(s)-W^{*}\right)$. Because $W^{*}>L(0)$, the same argument as in the proof of Lemma 4 applies.

Lemmas 2 to 5 complete the proof of Proposition 3.

We next show the upper and lower bounds of the licensor's revenue in the bargaining set $\tilde{M}^{N}$. It is completely characterized when the core is nonempty. A necessary and sufficient condition for the nonemptiness of the core is shown in Proposition 2. If $C^{N} \neq \emptyset, \tilde{C}^{N} \neq \emptyset$ by Lemma 1. $\tilde{C}^{N}$ is already characterized in Proposition 2.

Proposition 4 Let $x \in \tilde{M}^{N}$. Then, we have the following.

(a) If $n>s^{*}$, then $n(W(n)-L(0)) \leq x_{0} \leq s^{*}\left(W\left(s^{*}\right)-L(0)\right)$.

(b) If $n=s^{*}$, then $\tilde{M}^{N}=\tilde{C}^{N}$.

Proof: First, we show (a). Let $\bar{x}=L(0)+\epsilon_{1}$ where $\epsilon_{1}>0$. If $x_{0}=$ $n(W(n)-\bar{x})$, the licensor can make an objection $\left(y,\{0\} \cup S^{*}\right)$ such that $y_{i}>x_{i}$ for any $i \in\{0\} \cup S^{*}$, because $n(W(n)-\bar{x})=n(W(n)-L(0))-n \epsilon_{1}<$ $s^{*}\left(W\left(s^{*}\right)-L(0)\right)-s^{*} \epsilon_{1}=s^{*}\left(W\left(s^{*}\right)-\bar{x}\right)$. Because $\bar{x}>L(0)$, no counter objection can be made by any $i \in N \backslash S^{*}$, contradicting that $x \in \tilde{M}^{N}$. Thus, $\bar{x} \leq L(0)$. Lemma 2 completes the proof of (a).

Next, we show (b) with the following lemma.

Lemma 6 If $x \in \tilde{M}^{S^{*}}$, then $\bar{x} \geq L(0)$.

Proof : Let $x \in \tilde{M}^{S^{*}}$. Suppose $\bar{x}<L(0)$. Then, a licensee $i \in S^{*}$ has an objection $(y, N)$ against the licensor in $x$, such that $y_{k}=L(0)$ for any $k \in$ 
$N$. If the licensor had a counter objection $(z,\{0\} \cup R)$ to the objection $(y, N)$,

$$
\begin{aligned}
& r W(r) \geq z_{0}+\sum_{k \in R} z_{k}, \text { where } r=|R| \\
& z_{0} \geq x_{0}, \text { and } z_{k} \geq y_{k}=L(0) \text { for any } k \in R .
\end{aligned}
$$

Because $\bar{x}<L(0), x_{0}=s^{*} W\left(s^{*}\right)-s^{*} \bar{x}>s^{*} W\left(s^{*}\right)-s^{*} L(0)$. Hence,

$$
r W(r) \geq z_{0}+\sum_{k \in R} z_{k}>s^{*} W\left(s^{*}\right)-s^{*} L(0)+r L(0),
$$

which violates the definition of $s^{*}$. Thus, $i$ 's objection $(y, N)$ cannot be countered by the licensor, contradicting that $x \in \tilde{M}^{S^{*}}$.

Proposition 2 ensures $\tilde{C}^{N} \neq \emptyset$ if $s^{*}=n$. By the definitions, $\tilde{C}^{N} \subseteq \tilde{M}^{N}$. We show $\tilde{C}^{N} \supseteq \tilde{M}^{N}$. Let $x \in \tilde{M}^{N}$. Suppose that there is an $x \in \tilde{M}^{N}$ such that $x \notin \tilde{C}^{N}$. Because $x \in \tilde{M}^{N}, \bar{x} \geq L(0)$ by Lemma 6 . Because $x \notin \tilde{C}^{N}$, there is $\{0\} \cup T$ with $x_{0}+\sum_{i \in T} x_{i}<t W(t)$, where $t<n$. Let $(y,\{0\} \cup T)$ be an objection of the licensor against some $i \in N \backslash T$ in $x$ such that $y_{k}=x_{k}+\epsilon_{2}$ for any $k \in\{0\} \cup T$ and $(t+1) \epsilon_{2}=t W(t)-\left(x_{0}+\sum_{i \in T} x_{i}\right)>0$. Because $\bar{x} \geq L(0), i$ has no counter objection, contradicting that $x \in \tilde{M}^{S}$. Thus, $\tilde{M}^{N}=\tilde{C}^{N}$.

Let $S^{*}$ be a set $S \subseteq N$ with $|S|=s^{*}$. Proposition 3 (b) directly implies the next proposition.

Proposition 5 If $n / 2 \leq s^{*}<n$, then $\tilde{M}^{S^{*}}=\left\{x^{*}\right\}$, where

$$
x_{k}^{*}= \begin{cases}s^{*}\left(W\left(s^{*}\right)-L(0)\right) & \text { if } k=0 \\ L(0) & \text { if } k \in S^{*} \\ L\left(s^{*}\right) & \text { if } k \in N \backslash S^{*} .\end{cases}
$$

Proposition 5 says that, if the number of licensees that maximizes their total surplus is greater than the number of existing non-licensees, each symmetric bargaining set for a coalition structure is a singleton.

remark 4: In the linear example described in Section 2, there exists a threshold $\hat{\epsilon}$ of the cost reduction such that $n / 2 \leq s^{*}$ if and only if $\epsilon \leq \hat{\epsilon}$. It is easy to confirm that the number $s^{*}$ of firms increases as the patented cost-reducing technology becomes smaller in a Cournot market.

The next proposition ensures that $x^{*}$ is a stable profit sharing if $S=S^{*}$. 
Proposition $6 x^{*} \in \tilde{M}^{S^{*}}$.

Proof: Consider any objections $(y,\{0\} \cup T)$ of the licensor against a licensee $i \in S$ in $x^{*}(T \neq N, T \neq \emptyset)$. If $\sum_{k \in T} y_{k} \geq t L(0)$, we have $t W(t) \geq$ $y_{0}+\sum_{k \in T} y_{k}>x_{0}^{*}+t L(0)=s^{*}\left(W\left(s^{*}\right)-L(0)\right)+t L(0)$, which violates the definition of $s^{*}$. If $\sum_{k \in T} y_{k}<t L(0)$, firm $i$ can make a counter objection $(z, N)$ to the objection made by the licensor such that

$$
z_{i}= \begin{cases}L(0) & \text { if } k \in S \backslash T \\ y_{k}+\epsilon_{3} & \text { if } k \in T \\ L(0) & \text { if } k \in(N \backslash S) \backslash T,\end{cases}
$$

where $\epsilon_{3}=\left(t L(0)-\sum_{k \in T} y_{k}\right) / t>0$. Confirm that $\sum_{k \in N} z_{k}=n L(0)$, $z_{k} \geq x_{k}$ for any $k \in N$ and $z_{k}>y_{k} \forall k \in T$. Hence, no valid objection can be made by the licensor against any firms in $x^{*}$.

Next consider any objections $\left(u^{\prime}, R\right)$ of a firm $i \in S^{*}$ against the licensor in $x^{*}(0 \notin R)$. Let

$$
u_{k}^{\prime}= \begin{cases}u_{k} & \text { if } k \in R \\ x_{k}^{*} & \text { if } k \in N \backslash R .\end{cases}
$$

If $R \neq N, \sum_{k \in R} u_{k}<r L(0)$, where $r=|R|$. If $R=N, \sum_{k \in N} u_{k}=n L(0)$. Arrange the payoffs of all the firms in non-decreasing order. Take the first $s^{*}(<n)$ firms and let $Q$ be the set of them. Because $\sum_{k \in Q} u_{k}^{\prime} \leq s^{*} L(0)$, the licensor can make a counter objection to the objection. Hence, no valid objection can be made by any licensees against the licensor in $x^{*}$.

\subsection{The Optimal Number of Licenses: Implications}

This subsection considers the optimal number of licenses. First, we consider it as the licensor's choice at invitation stage (i) to complete our analysis. Next, we discuss the latest non-cooperative result by Sen and Tauman (2007) from our viewpoint.

Recall the bargaining outcomes at stage (ii) derived as our propositions. Lemma 2 implies that in any stable profit sharing the licensor can never obtain more than $s^{*}\left(W\left(s^{*}\right)-L(0)\right)$ as its revenue under any permissible coalition structures. On the other hand, Proposition 5 suggests that if 
$n / 2 \leq s^{*}<n$, the revenue of the licensor's is uniquely determined as $s^{*}\left(W\left(s^{*}\right)-L(0)\right)$, because $\tilde{M}^{S^{*}}$ is a singleton. Hence, if $n / 2 \leq s^{*}<n$ then the licensor should invite $s^{*}(<n)$ firms to the negotiation and license its patented technology to them. The stable profit sharing is completely specified in this case.

In the other cases, however, we cannot completely determine the optimal number of licenses, because $\tilde{M}^{S^{*}}$ may not be a singleton. Proposition 6 indicates that the licensor may obtain $s^{*}\left(W\left(s^{*}\right)-L(0)\right)$ as negotiation results, but it is not guaranteed. When $s^{*}=n$, in particular, it might be better for the licensor not to invite all the firms to the negotiation, if the collective bargaining power of firms is large; it might be better for it to invite $n-1$ firms to the negotiations if $(n-1)(W(n-1)-L(0))>n(W(n)-\bar{x})$ with $\bar{x}>L(0)$.

One may think we could specify the stable profit sharing in our generalized patent licensing game by characterizing the bargaining set for a coalition structure $P^{S^{*}}$ in the case of $1 \leq s^{*} \leq n / 2$. Unfortunately, we cannot obtain tighter predictions regarding the structure of the bargaining sets. (See the Further Characterization in the Appendix.)

Next, we briefly mention the optimal number of licenses derived as the latest non-cooperative result. In the linear example described in Section 2, Sen and Tauman (2007) suggest that, in the subgame perfect equilibrium, the licensor sell the license to at least $\hat{s}-1(n-1$ if $\hat{s}=n)$ firms $(n \geq 3)$ under the optimal combination of fee and royalty, where the fee is charged by means of auction, and that the net profit of every licensee is then the Cournot profit $L(\hat{s}-1, \delta)$ of a non-licensee. They seem to give an answer to the open question that has not been completed for about two decades because Kamien and Tauman $(1984,1986)$.

From our viewpoint, on the contrary, if every licensee is forced to receive such an extraordinarily low profit as suggested by Sen and Tauman, all the licensees may jointly "boycott" the license because they can communicate among them as emphasized in Remark 1. By doing so, every firm regains $L(0)$, which is its profit before the patented technology is developed. Truly, this coalitional deviation is not self-enforcing in any non-cooperative sense. Some firms that once rejected the license may breach the joint commitment 
on the deviation, each recontracting independently with the licensor. In such a case, however, every firm that wishes to recontract demands at least more than $L(\hat{s}-1, \delta)$ for its profit. Moreover, if the profit $L(0)$ is not guaranteed in recontracting, the joint commitment to reject the license may be robust. This is the point which is required of our solutions as well as coalition-proof Nash equilibrium (a noncooperative solution).

\section{$5 \quad$ Final Remarks}

Finally, we argue the following three points as final remarks. One is on characteristic functions, another is on the Shapley value studied in the related literature, and the other is on a solution for a more generalized model. The latter two remarks propose the topics for future research.

\subsection{Other Characteristic Functions}

The characteristic function we defined in Subsection 3.1 does not necessarily exhibit super-additivity often presumed in the cooperative analysis. We say that a characteristic function $v^{\prime}$ is super-additive if $v^{\prime}(S \cup T) \geq v^{\prime}(S)+$ $v^{\prime}(T)$ for all coalitions $S$ and $T$ with $S \cap T=\emptyset$. Super-additivity is the feature of characteristic functions required in analyzing how to distribute the total payoff in the grand coalition, because the grand coalition may not actually form without it. In fact, Aumann and Drèze (1974) did not require it for analysis of games with coalition structures, although they devoted one section to the super-additive cover of a characteristic function and its application to the core. ${ }^{16}$ It is, however, worth considering our characteristic function in comparison to other ones used in TW and DMN.

In this paper, we prohibit firms from forming any cartels in the market. This is the assumption under which we consider the same situation as the one in the non-cooperative analysis in the literature. A coalition is hence regarded as merely a group within which communication among its members is allowed. This is one of the reasons why our characteristic function does not necessarily satisfy super-additivity.

\footnotetext{
${ }^{16}$ See Section 10 in their paper.
} 
To confirm this, we note that in the linear example in Section 2, if the size of a coalition $\{0\} \cup S^{\prime}$ is not so large that $s^{\prime} \leq(n+1) / 2$ and $s^{\prime} \leq \hat{s}$, the values of our characteristic function are the same as the ones derived by TW's characteristic function, which is described as below. The firms in $\{0\} \cup S^{\prime}$ can merge into a single entity or can operate a few (or all) of the licensees and shut down the others. Suppose that $\{0\} \cup S^{\prime}$ operates $m$ licensees and shuts down $s^{\prime}-m$ firms, where $1 \leq m \leq s^{\prime}$. The complement, $N \backslash S^{\prime}$, can also operate some non-licensees, say $l$, where $1 \leq l \leq n-s^{\prime}$ and shut down the other $n-s^{\prime}-l$ firms. It is assumed that the $m+l$ active firms compete in the market $\grave{a} l a$ Cournot. Given $m$ and $l$, the Cournot profits of each licensee and each non-licensee are denoted by $W(m, l)$ and $L(m, l)$, respectively. TW defined the worth of coalition $\{0\} \cup S^{\prime}$ as the largest total Cournot profit of $\{0\} \cup S^{\prime}$ given the most offensive strategy of the complement:

$$
\tilde{v}\left(\{0\} \cup S^{\prime}\right)=\min _{1 \leq l \leq n-s^{\prime}} \max _{1 \leq m \leq s^{\prime}} m W(m, l) .
$$

The worth of coalition $S^{\prime}$ is defined in the same way. TW showed that the maxmin value coincides with the minmax value for any sets $S^{\prime}$ of firms. The maxmin or minmax approach itself is a well-known way to derive status quo points in two-person bargaining problems from non-cooperative games. This characteristic function $\tilde{v}$ exhibits the super-additivity. Thus, we can see that prohibiting any cartels in the market causes our characteristic function $v$ not necessarily to be super-additive. As noted in Section 1, however, we need this assumption to consider the same situation as those formalized with non-cooperative mechanisms in the literature from a practical viewpoint of patent licensing.

On the other hand, we can retain super-additivity even if firms cannot form any cartels. The characteristic function in DMN (applied to a game of information trading) has this property. In the context of patent licensing, a patented technology is licensed in the most efficient way among a seller and potential buyers of that technology, i.e.,

$$
\hat{v}\left(\{0\} \cup S^{\prime}\right)=\max _{0 \leq t \leq s^{\prime}} t W(t)+\left(s^{\prime}-t\right) L(t),
$$

where $W(0)=L(0)$. Note that $W(t)=W(t, n-t)$ and $L(t)=L(t, n-t)$ 
in the notation of TW. Let $t^{*}$ be the maximizer of $t W(t)+\left(s^{\prime}-t\right) L(t)$. According to this definition of $\hat{v}$, a patented technology is not necessarily licensed to all the potential buyers in coalition $S^{\prime}$, whereas $s^{\prime}-t^{*}$ non-buyers share the total profit of their coalition through sidepayments in reward for their cooperation for efficient sharing of a patented technology. They would, however, face a disadvantage when another patented technology is innovated after the game currently played; because they do not have the currently latest technology, they would suffer from lack of that technology even if newly innovated technology is licensed to them. So, we did not use $\hat{v}$.

\subsection{The Shapley Value}

Aumann and Drèze (1974) defined the Shapley value for a coalition structure as well as the other solutions and provided a set of axioms that characterizes the value. In the linear example in Section 2, we can find a case where the Shapley value for a coalition structure is not a stable profit sharing.

It is well known that the Shapley value is not necessarily in the core, but its relationship with the bargaining set has not been studied comprehensively. This is left for a future research.

\subsection{Limitation of Sidepayments}

We could have analyzed an alternative model where sidepayments are not allowed except payments to the licensor: for any $S \subseteq N$, each licensee $i \in\{0\} \cup S$ pays $p$ to the licensor as the upfront fee when licensed and there is no money transfer among firms in $S$. Even with these limited sidepayments, almost the same results are regained, so the presence of sidepayments does not an important role in our propositions.

With sidepayments, however, licensing by means of royalty only is not substantially different from licensing by means of upfront fee only. Hence, it is interesting to analyze the patent licensing game with limited sidepayments, which leads us to reconsider the traditional questions on "fee versus royalty" or "the optimal combination of upfront fee and royalty" from the original and practical viewpoint taken in this paper. We will show the complete results with limited sidepayments in another paper. 


\section{References}

[1] Aumann, R. J. and M. Drèze (1974) Cooperative games with coalition structures. Int. J. of Game Theory 3: 217-237

[2] Aumann, R. J. and M. Maschler (1964) The bargaining set for cooperative games, In: Advances in Game Theory, Dresher, M., L. S. Shaply and Tucker, A. W. (Eds.), Princeton University Press, 443-476

[3] Davis, M. and M. Maschler (1965) The kernel of a cooperative game. Naval Res. Logist. Quart. 12: 223-259

[4] Davis, M. and M. Maschler (1967) Existence of stable payoff configurations for cooperative games, In: Essays in Mathematical Economics in honor of Oskar Morgenstern, Schubik, M. (Ed.), Princeton University Press, 39-52 (abstract appeared in 1963 in Bulletin of American Mathematical Society 69, 106-108)

[5] Driessen, T., S. Muto, and M. Nakayama (1992) A cooperative game of information trading: the core, the nucleolus and the kernel. ZORMethods and Models of Operations Research 36: 55-72

[6] Kamien, M. I., S. S. Oren, and Y. Tauman (1992) Optimal licensing of cost-reducing innovation. J. Math. Econ. 21: 483-508

[7] Kamien, M. I. and Y. Tauman (1984) The private value of a patent: a game theoretic analysis. J. of Econ. Suppl. 4: 93-118

[8] Kamien, M. I. and Y. Tauman (1986) Fees versus royalties and the private value of a patent. Quart. J. Econ. 101: 471-491

[9] Kamien, M. I. Y. Tauman and I. Zhang (1988) Optimal license fees for a new product. Math. Soc. Sci. 16: 77-106

[10] Katz, M. L. and C. Shapiro (1985) On the licensing of innovation. Rand J. Econ. 16: 504-520

[11] Katz, M. L. and C. Shapiro (1986) How to license intangible property. Quart. J. Econ. 101: 567-589 
[12] Maschler, M. and B. Peleg (1966) A Characterization, existence proof and dimension bounds for the kernel. Pacific J. Math. 18: 289-328

[13] Muto, S. (1987) Possibility of relicensing and patent protection. Euro. Econ. Rev. 31: 927-945

[14] Muto, S. (1990) Resale-proofness and coalition-proof Nash equilibria. Games Econ. Behav. 2: 337-361

[15] Muto, S. (1993) On licensing policies in Bertrand competition. Games Econ. Behav. 5: 257-267

[16] Muto, S. and M. Nakayama, J. Potters and S. Tijs (1989) On Big Boss Games. Econ. Stud. Quart. 39: 303-321

[17] Nakayama M. and L. Quintas (1991) Stable payoffs in resale-proof trades of information. Games Econ. Behav. 3: 339-349

[18] Peleg, B. (1967) Existence theorem of for the bargaining set $\mathcal{M}_{1}^{i}$. In: Essays in Mathematical Economics in honor of Oskar Morgenstern, Schubik, M. (Ed.), Princeton University Press, 53-56 (the abstract appeared in 1963 in Bulletin of American Mathematical Society 69, 109-110)

[19] Schmeidler, D. (1969) The nucleolus of a characteristic function game. SIAM J. Appl. Math. 17: 1163-1170

[20] Sempere-Monerris, J. and J. Vannetelbosch (2001) The relevance of bargaining for the licensing of a cost-reducing innovation. Bull. Econ. Res. 53: 101-115

[21] Sen, D. (2005) Fee versus royalty reconsidered. Games Econ. Behav. 53: $141-147$

[22] Sen, D. and Y. Tauman (2007) General licensing schemes for a costreducing innovations. Games Econ. Behav. 59: 163-186

[23] Shapley, L. and M. Schubik (1967) Ownership and the production function. Quart. J. Econ. LXXXI: 88-111 
[24] Tauman, Y. and N. Watanabe (2007) The Shapley value of a patent licensing game: the asymptotic equivalence to non-cooperative results. Econ. Theory 30: 135-149

[25] Thrall, R. M., and W. F. Lucas (1963) n-person games in partition function form. Naval Res. Ligist. Quart. 10: 281-298

[26] Watanabe, N. and S. Muto (2006) Licensing agreements as bargaining outcomes: general results and two examples. Adv. Math. Econ. 8: 433447

[27] Watanabe, N. and Y. Tauman (2003) Asymptotic properties of the Shapley value of a patent licensing game. mimeo., Kyoto University

\section{Appendix}

\section{Proof of Lemma 1}

Lemma 1 For any $S \subseteq N$, there exists an $x \in \tilde{C}^{S}$ if $C^{S} \neq \emptyset$.

Proof: Let $y \in C^{S} \neq \emptyset$. Define $x \in \tilde{X}^{S}$ by $x_{j}=y_{j}$ if $j \notin S$ and $x_{i}=\bar{x}=$ $(1 / s) \sum_{i \in S} y_{i}$ if $i \in S$. For any $z \in X^{S}$, we write $\sum_{i \in S^{\prime} \subseteq\{0\} \cup N} z_{i}=z\left(S^{\prime}\right)$. Fix a coalition $T \subseteq\{0\} \cup N$ such that $T \cap S \neq \emptyset$. Let $l=|T \cap S|$. Then $\min _{U \subseteq S,|U|=l} y(U) \leq(l / s) y(S)=x(T \cap S)$. Hence,

$$
\begin{aligned}
& x(T)=x(T \backslash S)+x(T \cap S) \geq y(T \backslash S)+\min _{U \subseteq S,|U|=l} y(U) \\
& \geq \min _{U \subseteq S,|U|=l} y((T \backslash S) \cup U) \geq \min _{U \subseteq S,|U|=l} v((T \backslash S) \cup U)=v(T),
\end{aligned}
$$

because $y\left(S^{\prime}\right) \geq v\left(S^{\prime}\right)$ by $y \in C^{S}$ and $v((T \backslash S) \cup U)=v(T)$ for any $U \subseteq S$ with $|U|=l$ by the fact that all firms in $S$ are substitutes in $v$.

\section{Further Characterization}

We here try to find a more precise lower bound of the licensor's payoff $x_{0}$ in $\tilde{M}^{S^{*}}$ with $1 \leq s^{*} \leq n / 2$. (The other cases are shown in Propositions 4 and 5 , respectively.)

Let us begin with reconsidering the lower bound of the licensor's payoff $x_{0}$ in $\tilde{M}^{S}$. Given a coalition structure $P^{S}$ with $S \neq N$, define a payoff 
$x_{0}(t, r: S)$ for the licensor by

$$
\begin{aligned}
& x_{0}(t, r: S)=(t+r) W(t+r)-(t+r) L(0) \\
& \quad-(s-t)(L(0)-\bar{x})-(n-s-r)(L(0)-L(s)),
\end{aligned}
$$

where $T \subseteq S$ and $R \subseteq N \backslash S$. For any pairs of $T$ and $R$ with $r \geq 1$, let

$$
\bar{y}=L(0)+\frac{s}{r}(L(0)-\bar{x})+\frac{n-s-r}{r}(L(0)-L(s)) .
$$

When a vector $x \in \tilde{X}^{S}$ of payoffs with $x_{0}<x_{0}(t, r: S)$ is proposed, the licensor can make an objection $(y,\{0\} \cup(T \cup R))$ against a licensee $i \in S$ at $x$, where

$$
\begin{aligned}
& y_{0}=x_{0}+\epsilon_{4}, \quad y_{k}=\bar{x}+\epsilon_{4} \text { for any } k \in T, \\
& y_{k}=\bar{y}+\epsilon_{4} \text { for any } k \in R,
\end{aligned}
$$

and $\epsilon_{4}=\left(x_{0}(t, r: S)-x_{0}\right) /(t+r+1)$, if $\bar{y} \geq L(s)$, i.e., $s \bar{x} \leq s L(0)+(n-$ $s)(L(0)-L(s))$. Confirm that, for any $t$ and $r(\geq 1)$,

$$
\sum_{k^{\prime} \in\{0\} \cup(T \cup R)} y_{k^{\prime}}=(t+r) W(t+r)=v(\{0\} \cup(T \cup R)) .
$$

Then, whatever $T$ and $R$ are chosen, no counter objection $(z, N)$ can be made to the objection, because

$$
\begin{aligned}
& \sum_{k \in N} z_{k}=\sum_{k \in S \backslash T} x_{k}+\sum_{k \in T \cup R} y_{k}+\sum_{k \in(N \backslash S) \backslash R} x_{k} \\
& =(s-t) \bar{x}+(t+r) L(0)+(s-t)(L(0)-\bar{x}) \\
& \quad+(n-s-r)(L(0)-L(s))+(t+r) \epsilon_{4}+(n-s-r) L(s) \\
& =n L(0)+(t+r) \epsilon_{4}>n L(0)=v(N) .
\end{aligned}
$$

Next, we consider counter objections to $(y,\{0\} \cup T \cup R)$ made with the other coalitions. Consider a partition $[S,(N \backslash S) \backslash R, R]$ of $N$. Let $T^{\prime} \subseteq S$, $Q^{\prime} \subseteq(N \backslash S) \backslash R$ and $R^{\prime} \subseteq R$ such that $T^{\prime} \cup Q^{\prime} \cup R^{\prime} \neq N$. If, for any $\left(t^{\prime}, q^{\prime}, r^{\prime}\right)$,

$$
t^{\prime} \bar{x}+q^{\prime} L(s)+r^{\prime} \bar{y} \geq\left(t^{\prime}+q^{\prime}+r^{\prime}\right) L\left(\rho\left(t^{\prime}+q^{\prime}+r^{\prime}\right)\right),
$$

there is no counter objection that can be made by any licensees $i \in S$. Given a coalition structure $P^{S}$, denote by $\Theta(R)$ a set of all the $r$ 's each of which induces (3) under a partition $[S,(N \backslash S) \backslash R, R]$. Define a payoff $x_{0}\left(t^{*}, r^{*}: S\right)$ for the licensor by

$$
\begin{aligned}
& x_{0}\left(t^{*}, r^{*}: S\right)=\max _{t, r} x_{0}(t, r: S) \\
& \text { s.t. } 0 \leq t<s, r \in \Theta(R),
\end{aligned}
$$


where $t^{*}$ and $r^{*}$ are the maximizers of $x_{0}(t, r: S)$. There exists such a pair $\left(t^{*}, r^{*}\right) ; s\left(W(s)-W^{*}\right)=(n-s)(W(n-s)-L(0)) \leq x_{0}\left(t^{*}, r^{*}: S\right)$ in the case of $1 \leq s \leq n / 2$, and $s(W(s)-L(0)) \leq x_{0}\left(t^{*}, r^{*}: S\right)$ in the case of $n / 2 \leq s<n$, as shown in Lemmas 3,4 and 5 (and the definition of $W^{*}$ ).

We are now ready to state the next proposition by the definition of $x_{0}\left(t^{*}, r^{*}: S^{*}\right)$. Lemma 5 and Proposition 6 jointly show that the upper limit of $x_{0}$ in $\tilde{M}^{S^{*}}$.

Proposition 7 If $x \in \tilde{M}^{S^{*}}$ with $1 \leq s^{*} \leq n / 2$, then

$$
x_{0}\left(t^{*}, r^{*}: S^{*}\right) \leq x_{0} \leq s^{*}\left(W\left(s^{*}\right)-L(0)\right) .
$$

Confirm that the complete characterization of $\tilde{M}^{S^{*}}$ does not provide any tighter predictions regarding the structure of the bargaining set for a coalition structure in comparison to those shown by other propositions. 